\title{
EDITORIAL
}

\section{Research Integrity}

Enoka Corea (Dhttp://orcid.org/0000-0003-1450-2098, Himani Molligoda (Dhttp://orcid.org/0000-0002-9216-9035, Co-Editors

\section{Keywords: Research Integrity}

This is an open-access article distributed under a Creative Commons Attribution-Share Alike 4.0 Internationa License (CC BY-SA 4.0), which permits unrestricted use, distribution, and reproduction in any medium,

provided the original author and source are attributed and materials are shared under the same license.

Research ethics has traditionally focused on preventing exploitation of the subjects of research. Ethics Review Committee (or Institutional Review Board) approval is mandatory to conduct and publish research on human or animal subjects. However, ethical research is more than just ensuring the protection and welfare of the research participant. There is also the requirement to conduct and report research in an intellectually honest manner. This is what is known as research integrity.

Research integrity cannot be assured or monitored by an ERC and is solely dependent on the competence and trustworthiness of the researcher. However, it seems that many researchers are not sensitized to this concept. There are many breaches in research integrity, some of which are detected and resulting in retractions of published papers and discredit for the authors.

What, then, are the key requirements necessary to ensure that a research is conducted with scientific integrity?

\section{Ethical conduct of research}

The research objectives and study design should be drafted following a thorough literature search, to ensure adequate justification and precise study design. Peer review of the draft research protocol is ideal.

The researcher (and any co-investigators or research assistants) should have the necessary qualifications and competence and should free up adequate time to conduct the study to the highest possible standard.

All potential co-authors should share the responsibilities of the research.

Best research practices should be followed including good clinical practice (GCP) guidelines, good laboratory practice (GLP) guidelines and good manufacturing practice (GMP) guidelines.

All aspects of the methodology should be meticulously recorded and retained for an adequate period of time for inspection.

All research funds should be accounted for using standard accounting practices.

Researchers should comply with available national, professional and institutional policies, laws, regulations, guidelines and procedures. 
The research should be conducted and reported with the highest standards of objectivity, transparency and independence, sans bias, fabrication, falsification, exaggeration, misrepresentation or misinterpretation.

Any potential conflicts of interest should be declared and managed.

Researchers should respect the intellectual property rights of others and cite the previous work of other researchers when used. All substantial contributors to the research should be included in the publication and others who have facilitated the research, including the sponsor and the institution, should be acknowledged.

\section{Conclusion}

These 'other' ethical duties and responsibilities of the researcher are usually not given adequate publicity among young researchers during their training. Lack of knowledge could lead to breach of these ethical principles and loss of a researcher's reputation and the reputation of the institution where the research was conducted. The credibility of scientists, as a whole, will also be at stake. Therefore, it is imperative that training courses on research include these areas.

\section{References}

1. National Science and Technology Commission. A Guidebook on Research Ethics, Sri Lanka. (2004)

2. Ethical Guidelines for Biomedical Research on Human Subjects. Indian Council of Medical Research (2000)

3. National Ethical Guidelines for Health Research in Nepal. Nepal Health Research Council, Nepal (2001)

4. Guidelines on Ethical Conduct for Medical and Dental Practitioners registered with the Sri Lanka Medical Council. Sri Lanka Medical Council (2003) 